\title{
EL RETO DEL SOFTWARE LIBRE EN ESPAÑA
}

\section{THE CHALLENGE OF FREE SOFTWARE IN SPAIN}

Reynaldo Cordero: PAS Servicios Informáticos. Universidad de Alcalá de Henares (Madrid-España).

reynaldo.cordero@uah.es

\section{CURRÍCULUM VITAE}

Es funcionario de la Universidad de Alcalá de Henares. Representante de CSIT Unión Profesional.

\section{RESUMEN}

España no está siendo firme con los procedimientos de buena práctica en eAdministración, tal y como los recoge el propio Comisario Europeo para la Sociedad de la Información, Erkki Liikanen, en el simposio correspondiente. El tiempo pasa y puede llegar a ser demasiado tarde para España. Si el Ministerio quiere cumplir los plazos debería empezar por realizar un estudio detallado para después elaborar un plan de pruebas piloto, crear una "Agencia Estatal de Estudio e Incentivación del Software Libre" y acabar con la redacción y firma de un Convenios de cooperación entre entidades y asociaciones representativas del software libre en España y la Administración.esumen del artículo en idioma original (Book antiqua. 12. Minúsculas. Justificado. No sangrado). 


\section{PALABRAS CLAVE}

Administración - Sociedad de la Información - Software Libre

\section{ABSTRACT}

Spain is not being firm with the procedures of good practice in e-Government as the Commissioner collects the European Information Society, Erkki Liikanen, the corresponding symposium. Time passes and can be too late for Spain. If the Ministry wants to meet deadlines should start a detailed study and then develop a pilot plan to create a "State Agency and Incentives Study of Free Software" and end with the drafting and signing of cooperation agreements between entities and associations representing the free software and Management in Spain.

\section{KEY WORDS}

e-Government - Information Society - Free Software

\section{ÍNDICE}

1. Recomendaciones al Ministerio de Ciencia y Tecnología

2. ¿Cuándo sería demasiado tarde para España?

3. Enlaces sobre Europa

4. Objetivos 


\section{TEXTO:}

\section{Recomendaciones al Ministerio de Ciencia y Tecnología}

En Europa los distintos gobiernos se están tomando muy en serio el fenómeno del Software Libre, especialmente Francia, Alemania, Reino Unido y Finlandia, y esto se demuestra porque:

-Encargan detallados informes oficiales sobre el Software Libre.

-Crean agencias estatales de estudio e incentivación del Software Libre.

-Crean un número significativo de centros piloto donde introducen distintos tipos de experiencias con Software Libre.

-Colaboran estrechamente con asociaciones representativas del sector del software libre.

Respecto a la Unión Europea, la situación es la siguiente:

España no está siendo firme con los procedimientos de buena práctica en eAdministración, tal y como los recoge el propio Comisario Europeo para la Sociedad de la Información, Erkki Liikanen(1), en el simposio correspondiente (2), celebrado el 30 de junio pasado, en Luxemburgo, donde advierte que hablar de e-Administración supone hablar de formatos electrónicos estándares, y que no es adecuado que el ciudadano tenga que comprar un determinado software comercial para comunicarse o informarse con la Administración.

En este contexto, apoyándose en el Programa IDA (Intercambio de Datos entre Administraciones) de la Unión Europea, la Comisión está realizando un estudio -en colaboración con los estados miembros- sobre los programas de código abierto. Lamentablemente España no muestra una actitud decidida (3). 
El software de código abierto, prosigue Liikanen, es importante también por razones de seguridad, dado que el concepto de "seguridad mediante oscuridad" es un concepto obsoleto que mediante software de código abierto se puede superar. En aplicaciones de seguridad críticas se necesita de sistemas integrales con software libre.

La Comisión tiene que tener otras opciones en cuenta [aparte de la representada por la multinacional norteamericana Microsoft] y debe, en palabras de Liikanen, considerar la importancia creciente del software libre y de los estándares abiertos.

Por último, Liikanen vuelve a insistir en que "el software abierto puede crear nuevas oportunidades". Tenemos que conseguir que nuestro país haga un esfuerzo para que se realicen experiencias significativas suficientes para no quedar descolgados en el caso, ciertamente muy probable, de que nuestro entorno se pase al software abierto.

\section{2. ¿Cuándo sería demasiado tarde para España?}

Basta con leer textualmente el primer párrafo del epígrafe "Plazos" del "Plan de acción e-Europa"(4) para hacernos una idea:

Uno de los factores clave que impulsa la iniciativa e-Europa es la necesidad de llevar a cabo actuaciones urgentes dentro de plazos muy justos en campos críticos para la nueva economía. El enfoque en que se basa este plan de acción es centrarse en estas actuaciones y, de este modo, asegurar la rápida eliminación de las barreras que todavía existen. Por eso, establece una fecha clave, 2002, en la que deberán haberse alcanzado todos los objetivos. Si Europa no puede lograr los cambios necesarios rápidamente será demasiado tarde para alcanzar los ambiciosos objetivos de la cumbre de Lisboa. 


\section{Enlaces sobre Europa}

1) Comisión Europea. Comisario Erkii Likanen de Empresa y Sociedad de la Información.

http://europa.eu.int/comm/commissioners/liikanen/index_en.htm

2) Comisión E. Buenas prácticas en e-Administración. Procedimientos.

http://europa.eu.int/comm/di/symposium2000/liikanen/index.htm

3) Comisión E. Utilización de software de código abierto en el sector público. Objetivos 2001. Plan de acción para una Internet barata, segura y rápida.

$\underline{\text { http://europa.eu.int/information_society/eeurope/action_plan/stimulate/egov/m }}$ ember_states/print_en.htm

4) Comisión E. eEurope 2002. Plan de acción preparado por el Consejo y la Comisión Europea para el Consejo Europeo de Feira. Bruselas, 14/6/2000

http://europa.eu.int/information_society/eeurope/action_plan/pdf/actionplan_es. pdf

\section{Objetivos}

De interés para el Ministerio de Ciencia y Tecnología, para poder asimilar los retos y oportunidades que le brindaría el software libre: 
a) Realización de un estudio detallado (legal, económico, estratégico) sobre el impacto de la introducción de software libre en la Administración, a fin de considerar con seriedad su posible implantación en la misma.

b) Elaboración de un plan de pruebas piloto suficientemente extenso y bien diseñado, ya sea a nivel de la Administración, en la Universidad o mediante incentivos a la iniciativa privada, que permita caracterizar con precisión los factores que intervienen en el proceso de migración y de adaptación desde un entorno de software propietario a un nuevo modelo con software libre.

c) Creación de una "Agencia Estatal de Estudio e Incentivación del Software Libre", al estilo francés o alemán. De forma alternativa o simultánea se debería crear un "Observatorio del Software Libre", con expertos de la Administración y del mundo del software libre, para que sirva como referencia y asesoramiento reconocido por parte de la Administración.

d) Redacción y firma de un Convenios de cooperación entre entidades y asociaciones representativas del software libre en España y la Administración para la elaboración de estudios y la coordinación de asuntos de interés comunes (Por ejemplo, con HispaLinux). 